International Journal of Modern Physics A

(C) World Scientific Publishing Company

\title{
Threshold Hyperon Production at COSY-11
}

\author{
T. ROŻEK, D. GRZONKA, K. KILIAN, P. KOWINA, W. OELERT, T. SEFZICK, \\ P. WINTER, M. WOLKE, P. WÜSTNER \\ IKP and ZEL Forschungszentrum Jülich, D-52425 Jülich, Germany \\ M. SIEMASZKO, W. ZIPPER \\ Institute of Physics, University of Silesia, Katowice 40-00\%, Poland \\ R. CZYŻYKIEWICZ, M. JANUSZ, L. JARCZYK, B. KAMYS, P. KLAJA, P. MOSKAL, \\ C. PISKOR-IGNATOWICZ, J. PRZERWA, J. SMYRSKI \\ Nuclear Physics Department, Jagellonian University, Cracow 30-059, Poland
}

H.H. ADAM, A. KHOUKAZ, R. SANTO, A. TÄSCHNER

IKP, Westfäche Wilhelms-Universität, D-48149 Münster, Germany

A. BUDZANOWSKI

Institute of Nuclear Physics, Cracow 31-342, Poland

Received (Day Month Year)

Revised (Day Month Year)

\begin{abstract}
The $\Lambda, \Sigma^{0}$ and $\Sigma^{+}$hyperon production in NN collisions is studied at the COSY - 11 installation in order to investigate the production mechanism as well as to extract information about the $\mathrm{Y}-\mathrm{N}$ interaction.
\end{abstract}

Keywords: hyperon production, $\mathrm{Y}-\mathrm{N}$ interaction

COSY-11 is an internal magnetic spectrometer experiment at the COoler SYnchroton and storage ring COSY in Jülich. It is equipped with scintillator hodoscopes and drift chambers for charged particle detection 1 and a scintillator/lead sandwich detector for neutrons 2 .

The $\Sigma^{0}$ and $\Lambda$ hyperon production near the kinematical threshold was studied by the COSY-11 collaboration in $p p \rightarrow p K^{+} \Lambda / \Sigma^{0}$ reactions. Data points, 16 for the $\Lambda$ and 13 for the $\Sigma^{0}$ channel, were taken in the excess energy range between $0.68 \mathrm{MeV}$ and $59.3 \mathrm{MeV}$ for the $\Lambda$ hyperon and between $2.8 \mathrm{MeV}$ and $59.1 \mathrm{MeV}$ for $\Sigma^{0} 345$. The cross section ratio $\sigma\left(p p \rightarrow p K^{+} \Lambda\right) / \sigma\left(p p \rightarrow p K^{+} \Sigma^{0}\right)$ below excess energies of $15 \mathrm{MeV}$ was measured to be around 28 in contrast to the value of about 2.5 determined for excess energies higher than $Q=300 \mathrm{MeV}$ [ The ratio for higher energies is in good agreement with the $\Lambda / \Sigma^{0}$ isospin relation, which is 3 (see figure 1). 


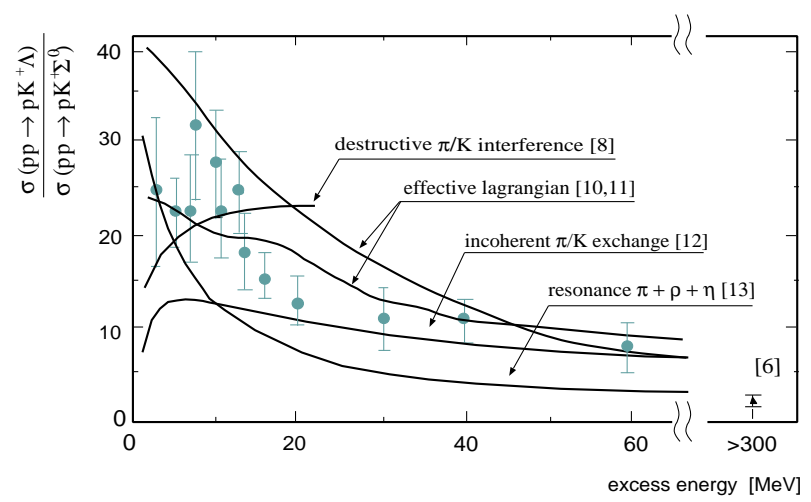

Fig. 1. Cross section ratio for $\Sigma^{0}$ and $\Lambda$ production in the threshold region. The data are compared to different models descriptions.

To explain this unexpected threshold behaviour, various theoretical scenarios within meson exchange models were proposed. Calculations have been performed with pion and kaon exchange added coherently $[89$ or incoherently 12 , including the excitation of nucleon resonances 101311 and heavy meson exchange $(\rho, \omega$ and $K^{*}{ }^{1011}$. Although the various descriptions differ even in the dominant basic reaction mechanism, all more or less reproduce the trend of an increase in the cross section ratio in the threshold region. The present data are not sufficient to definitely exclude possible explanations. Further studies e.g. for the other isospin projections will help to understand the threshold hyperon production. To be more specific lets consider the Jülich meson exchange mode $[89$ where calculations are available for other $\Sigma$ channels. Within this model the large cross section ratio $\sigma(\Lambda) / \sigma\left(\Sigma^{0}\right)$ at threshold is reproduced by a destructive interference of $\pi$ and $K$ exchange amplitudes. Calculations of the $\Sigma^{+}$production in this model predict a factor of three higher cross section compared to the $\Sigma^{0}$ channel for a destructive and a factor of three lower for a constructive interference, a clear experimentally accessible signal. The ratio between $\Sigma^{+}$and $\Sigma^{0}$ production will also differ strongly if the dominant production mechanism runs via an intermediate $N^{*}$ excitation or not.

Recently the $\Sigma^{+}$production was measured at the COSY-11 installation via $p p \rightarrow n K^{+} \Sigma^{+}$at $\mathrm{Q}=13 \mathrm{MeV}$ and $\mathrm{Q}=60 \mathrm{MeV}$. The $\Sigma^{+}$is identified via the missing mass technique, by detecting the remaining reaction products $-K^{+}$and neutron. The experimental resolution of the missing mass determination depends on the reconstruction accuracy of the four-momentum vectors for the registered neutrons and kaons. The momentum vector of the $K^{+}$meson can be established by tracking the $K^{+}$trajectory reconstructed from signals registered in the drift chambers (D1 and D2 in the figure 2) through the magnetic field back to the target point. Assuming a hit in the neutron detector being due to a neutron, the fourmomentum vector of the neutron is given by the measured velocity, the direction 


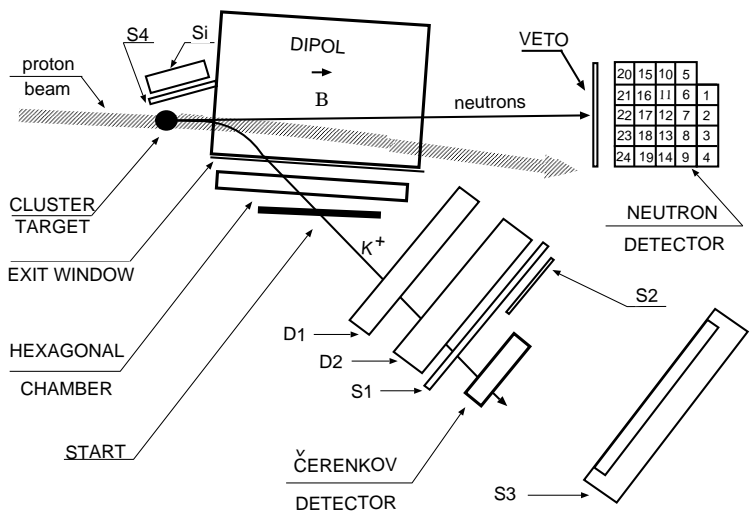

Fig. 2. COSY-11 detection setup with the superimposed tracks of kaon and neutron from the $p p \rightarrow n K^{+} \Sigma^{+}$reaction.

(given by the first hit module) of the neutron which can be reconstructed with an accuracy of at least the size of the module $\left(9 \times 9 \mathrm{~cm}^{2}\right)$ and the known mass. The background from charged particles hitting the neutron detector is discriminated by veto scintillators (VETO in figure 2).

From the Monte Carlo studies the identification of the $\Sigma^{+}$events is expected to be comparable to the $\Sigma^{0}$ or $\Lambda$ channels? The data are presently under analysis.

\section{Acknowledgments}

The work has been supported by the European Community - Access to Research Infrastructure action of the Improving Human Potential Programme, by the DAAD Exchange Programme (PPP-Polen), by the Polish State Committe for Scientific Research (grants No. 2P03B07123 and PB1060/P03/2004/26) and by the Research Centre Jülich.

\section{References}

1. S. Brauksiepe et al., Nucl. Inst.\& Meth. A376, 397-410 (1996).

2. P. Moskal et al., nucl-ex/0311003

3. J.T. Balewski et al., Phys.Lett. B420, 211 (1998).

4. S. Sewerin et al., Phys. Rev. Lett. 83, 682 (1999).

5. P. Kowina et al., e-Print Archive nucl-ex/0402008 Eur. Phys. J. A, in print.

6. A. Baldini et al., Total Cross-Section for Reactions of High-Energy Particles, (Springer, Berlin, 1988).

7. T. Rożek et al., COSY Proposal no. 117 (2002).

8. A. Gasparian et al., Phys. Lett. B480, 273 (2000).

9. A. Gasparian et al., Nucl.Phys. A684, 397-399 (2001).

10. R. Shyam et al., Phys. Rev. C63, 022202 (2001).

11. R. Shyam hep-ph/0406297

12. A. Sibirtsev et al., Nucl.Phys. A646, 427-443 (1999).

13. A. Sibirtsev et al., nucl-th/0004022 v2 (2000). 